\begin{tabular}{|l|l|}
\hline $\begin{array}{l}\text { Instituto de } \\
\text { Geriatria e Gerontologia }\end{array}$ & $\begin{array}{l}\text { Pan American Journal of Aging Research } \\
\text { PAJAR, Porto Alegre, v. 8, p. 1-10, jan.-dez. } 2020 \\
\text { ISSN-L: 2357-9641 }\end{array}$ \\
\hline http://dx.doi.org/10.15448/2357-9641.2020.1.37479 & \\
\cline { 2 - 3 }
\end{tabular}

SEÇÃO: ARTIGO ORIGINAL

\title{
Avaliação multidimensional da fragilidade em idosos hospitalizados
}

Multidimensional assessment of frailty in hospitalized elderly

Evaluación multidimensional de la fragilidad en ancianos hospitalizados

\author{
Luciano Alves Leandro ${ }^{1}$ \\ orcid.org/0000-0002-5073-1237 \\ luciano.leandro@pucpr.br
}

\section{Laryssa Mireli \\ Rodrigues Gomes ${ }^{1}$}

orcid.org/0000-0003-0502-9964

laryssarodrigues.mireli@hotmail.com

\section{Juliane Pessoa \\ Chevônica ${ }^{1}$ \\ orcid.org/0000-0001-7014-944X \\ juliane.pessoa@outlook.com}

Recebido em: 29/3/2020.

Aprovado em: 29/7/2020

Publicado em: 30/10/2020.

\section{Resumo}

Introdução: a Sindrome da Fragilidade (SF) pode representar um impacto significativo nos cuidados hospitalares aos idosos gerando desfechos com prognósticos funcionais variados.

Objetivo: conhecer a multidimensionalidade envolvida no processo de hospitalização de idosos e a sua condição de fragilidade.

Métodos: estudo transversal com abordagem descritiva que avaliou 150 idosos com 60 anos ou mais, internados em dois grandes hospitais na cidade de Curitiba, PR. Foram coletados dados relacionados à caracterização sociodemográfica; fatores cognitivos pelo Mini Exame do Estado Mental (MEEM); nivel de fragilidade pela Edmonton Frail Scale (EFS); depressão pela Escala de Depressão Geriátrica (EDG-15); e força de preensão manual (FPM) verificada por dinamometria manual. Resultados: a média de idade foi de 70,0 77,9 anos e $92(61,3 \%)$ eram homens. $30 \%$ dos idosos foram classificados como vulneráveis, $27,3 \%$ evoluindo como uma fragilidade leve, $22,7 \%$ moderada, 9,3\% considerada uma forma grave e, apenas $10,7 \%$ normais. Fatores como diminuição de força de preensão palmar e depressão foram estatisticamente associados à condição de fragilidade, servindo como parâmetros analíticos dentro de uma multidimensionalidade avaliada. Conclusão: os colaboradores deste estudo apresentam-se mais vulneráveis, tanto físico como cognitivamente, levando a um cenário condizente a uma redução de suas habilidades motoras, pior expectativa de vida e consequente morte precoce. Palavras-chave: idoso fragilizado, hospitalização, perfil de saúde.

\section{Abstract}

Introduction: a Fragility Syndrome (SF) can have a significant impact on hospital care with the elderly, generating outcomes with varied functional prognoses.

Objective: know the multidimensionality involved in the process of hospitalization of elderly and their condition of fragility.

Methods: a cross-sectional study with a descriptive approach, which evaluated 150 elderly patients aged 60 years old or older hospitalized in two large hospitals in the city of Curitiba, Paraná state. Data related to sociodemographic characterization data were collected; cognitive factors by Mini Mental State Examination (MMSE): level of fragility by the Edmonton Frail Scale (EFS); depression by the Geriatric Depression Scale (EDG-15); manual grip force (FPM) verified by manual dynamometry. Results: the mean age was $70.0 \pm 7.9$ years old and $92(61.3 \%)$ were men. $30 \%$ of the elderly were classified as vulnerable, $27.3 \%$ were evolving towards mild fragility, $22.7 \%$ classified as moderate and $9.3 \%$ were considered a severe form and only $10.7 \%$ normal. Factors such as decreased palmar grip strength and depression were statistically associated with the fragility condition, serving as analytical parameters within a multidimensionality assessed.

Conclusion: the study collaborators are more vulnerable, both physically and cognitively, leading to a scenario consistent with reduced motor skills, worse life expectancy and consequent early death.

Keywords: frail elderly, hospitalization, health profile. 


\section{Resumen}

Introducción: el Sindrome de la Fragilidad (SF) puede representar un impacto significativo en el cuidado hospitalario a los ancianos generando resultados con pronósticos funcionales variados.

Objetivo: conocer la multidimensionalidad involucrada en el proceso de hospitalización en ancianos y su condición de fragilidad.

Métodos: estudio transversal con abordaje descriptivo, que evaluó 150 ancianos con 60 años o más, internados en dos grandes hospitales en la ciudad de Curitiba-PR. Se recogieron datos relacionados con la caracterización sociodemográfica; factores cognitivos travésel Mini Examen del Estado Mental (MEEM); nivel de fragilidad por Edmonton Frail Scale (EFS); depresión por la Escala de Depresión Geriátrica (EDG-15); fuerza de asimiento manual (FPM) comprobada por dinamometría manual. Resultados: el promedio de edad fue de 70,0 \pm 7.9 años y 92 (61,3\%) eran hombres. El 30\% de los ancianos fueron clasificados como vulnerables, el 27,3\% evolucionó como una fragilidad leve, el $22,7 \%$ moderada y el 9.3\% considerado una forma grave y sólo el 10,7\% normal. Factores como disminución de fuerza de asimiento palmar y depresión fueron estadísticamente asociados a la condición de fragilidad, sirviendo como parámetros analíticos dentro de una multidimensionalidad evaluada.

Conclusión: los colaboradores de este estudio se presentan más vulnerables, tanto físicos como cognitivamente, llevando a un escenario acorde a una reducción de sus habilidades motoras, peor expectativa de vida y consecuente muerte precoz.

Palabras clave: ancianos fragilizados, hospitalización, perfil de salud.

\section{Introdução}

Estimativas projetam um número de idosos superior a 30 milhões até 2025 e a velhice poderá ser tanto acompanhada por altos niveis de doenças crônicas quanto por saúde e bem-estar. Tal aumento colocará o Brasil, em 2025, como a sexta população de idosos do mundo. ${ }^{1}$

Diante dessa expectativa, surge o "idoso frágil", uma consequência do envelhecimento relacionada ao processo de cronicidade e o contexto mais propício para definir a fragilidade é descrito por Fried et al. ${ }^{2}$ como um declínio de energia em espiral, com diminuição das reservas fisiológicas e aumento do déficit funcional, associados às mudanças físicas, causado por alterações fisiológicas dos sistemas musculoesquelético, neuroendócrino e imunológico com foco na multidimensionalidade, caracterizada por vulnerabilidade a estressores físicos, psicológicos e sociais. ${ }^{3}$
Fried et al. ${ }^{2}$ propõe a identificação bem como o diagnóstico da Síndrome da Fragilidade (SF) de acordo com cinco critérios: 1) perda de peso não intencional; 2) exaustão avaliada por autorrelato de fadiga; 3) diminuição da força de preensão manual; 4) baixo nível de atividade física; e 5) diminuição da velocidade de caminhada. Tais mudanças provocam efeitos adversos, como quedas, hospitalização, institucionalização, declínio funcional e morte precoce.

De acordo com dados estatísticos apresentados pelo DATASUS, 4 no ano de 2009 foram registradas 2.332.747 internações hospitalares de individuos com idade superior a 60 anos, que corresponderam a $21 \%$ das admissões hospitalares.

Fatores relacionados à hospitalização como um estado de maior vulnerabilidade, baixa reserva homeostática e uma menor capacidade de responder aos diferentes tipos de estresse ${ }^{2}$ podem caracterizar um dos resultados da condição de fragilidade comumente relatados na literatura ${ }^{5} \mathrm{e}$ podem influenciar negativamente as mudanças entre as condições pós-alta. ${ }^{6}$

Buscas frequentes por serviços de saúde resultam em uma maior ocupação de leitos nos hospitais pela população idosa, pois o idoso consome mais serviços de saúde, as internações hospitalares são mais frequentes e o tempo de ocupação do leito é maior quando comparado a outras faixas etárias. Esse fato é decorrência do padrão das doenças dos idosos, que são crônicas e múltiplas, exigem acompanhamento constante, cuidados permanentes, medicação contínua e exames periódicos, ${ }^{7}$ condições que podem levarà SF. ${ }^{6}$ Essa sindrome pode representar um impacto significativo nos cuidados hospitalares com ido$\operatorname{sos}^{2}$ em relação ao estado funcional, dificultando a recuperação de condições de fragilidade e/ou pré-fragilidade e estão, ainda, fortemente associadas a quadros de vulnerabilidade e a maiores indices de mortalidade precoce. ${ }^{8}$

Além do processo de fragilização, custos econômicos e a hospitalização do idoso, diversas consequências vão atingir diretamente a fun- 
cionalidade, de maneira que, para a maioria dos idosos, esse evento pode ser visto como um fator que corrobora com um consequente aumento das taxas de morbimortalidade..$^{9,10}$

Com o impacto de toda a transformação epidemiológica que o envelhecimento populacional encontra, a prevalência de idosos doentes crônicos alcança cerca de $60 \%{ }^{11}$ e as necessidades de saúde, principalmente expressas pela cronicidade das doenças, respondem pelos principais determinantes da hospitalização quando falamos na população idosa. ${ }^{12}$ Assim, é crescente o interesse na avaliação multidimensional dos idosos e nas implicações que a multimorbidade desencadeia para organização e oferta de ações e serviços de saúde. ${ }^{13}$

Maiores riscos de complicações fisiológicas decorrentes das doenças ${ }^{14}$ em comparação aos resultados de diferentes pontos de corte para multimorbidade mostraram que diagnósticos de três ou mais doenças foi um indicador de grande impacto na predição de hospitalização, reforçando o uso deste ponto de corte na avaliação da multimorbidade em idosos ${ }^{15}$. Dessa forma, quanto maior o número de doenças, maior a probabilidade de internações, o que representa desafios importantes para a atenção aos idosos no Brasil.

Tendo em vista a crescente carência de demandas assistenciais associada a um crescimento populacional, com questões relacionadas à qualidade dos serviços de saúde, o objetivo deste estudo foi conhecer a multidimensionalidade envolvida no processo de hospitalização em idosos e sua condição de fragilidade.

\section{Método}

Este estudo foi aprovado pelo Comitê de Ética em Pesquisa da Pontifícia Universidade Católica do Paraná (CEP-PUCPR), com aprovação e parecer 1.955.798/2017 do dia 9 de março de 2017 . estando de acordo com a resolução 466/12 do Conselho Nacional de Saúde.

Trata-se de um estudo transversal com uma abordagem descritiva, ${ }^{16}$ realizado em dois grandes hospitais na cidade de Curitiba, PR, Brasil, de março de 2017 a março de 2018. A coleta de dados ocorreu no período de abril a setembro de 2017 . por meio de entrevistas, observação e registro de informações, utilizando-se instrumentos específicos de coleta de dados elaborados especificamente para esse estudo. Não foi realizado cálculo amostral, e sim, uma amostra de conveniência, uma vez que foram incluídos no estudo todos os idosos que permaneceram internados nas Instituições Hospitalares em questão, no periodo de tempo referido, por, no mínimo, 24 horas.

As avaliações foram aplicadas pelos pesquisadores, devidamente orientados para tal, de modo a garantir a homogeneidade da coleta, bem como para conscientizar os participantes da finalidade da avaliação e da utilização dos resultados.

A população foi composta por idosos que atenderam aos seguintes critérios de inclusão: idade igual ou superior a 60 anos; com cognitivo preservado segundo a escala Mini Exame do Estado Mental (MEEM); internados há mais de um dia. Foram excluidos desse estudo idosos que apresentassem funções cognitivas que impossibilitassem responder aos instrumentos da pesquisa; amputação de membros superiores e/ou membros inferiores; e internados em unidades fechadas (emergência, sala de recuperação, UTI, centro cirúrgico e unidade de estudo hemodinâmico).

Dos 163 colaboradores, 4 se recusaram a participar e 9 não atenderam aos critérios de inclusão, resultando em 150 participantes.

Foi realizado um rastreamento por meio do MEEM, teste cognitivo breve composto por itens a respeito de orientação espaço-temporal, registro, memória de curto prazo, atenção, cálculo, linguagem e praxia construcional, possibilitando uma avaliação concisa do estado mental de um paciente. O escore varia de o a 30 e para a correção não são considerados erros gramaticais ou ortográficos, considerando-se apenas a escolaridade do paciente. Para idosos com baixa escolaridade, utilizam-se os seguintes pontos de corte: 20 para analfabetos, 25 para pessoas com um a quatro anos de escolaridade, 26 para 
aquelas com cinco a oito anos de escolaridade, 28 para individuos que estudaram de nove a onze anos e 29 para aqueles com escolaridade superior a onze anos. ${ }^{17}$ Logo após, seguiu-se com a caracterização demográfica do idoso, no qual se coletavam elementos de identificação como: nome; idade; sexo; escolaridade; estado civil; tempo de internamento; e prática de atividade física.

Em seguida foi realizada a avaliação de fragilidade, por meio da Edmonton Frail Scale (EFS), considerado um instrumento robusto pela capacidade de avaliar o idoso de forma multidimensional, traduzida e validada na língua portuguesa, composta por nove domínios (cognição, estado de saúde, independência funcional, suporte social, uso de medicação, nutrição, humor, continência urinária e desempenho funcional), distribuidos em 11 itens. Sua pontuação máxima é 17 e representa o nível mais elevado de fragilidade. Os escores para a análise da fragilidade são: 0-4, normais; 5-6, aparentemente vulnerável; 7-8, fragilidade leve; 9-10, fragilidade moderada; 11 ou mais, fragilidade severa. ${ }^{18}$

Foi realizada, também, uma avaliação afetiva por meio da Escala de Depressão Geriátrica (EDG15). ${ }^{19}$ Essa escala detecta sintomas depressivos no idoso através de 15 perguntas negativas/ afirmativas. O resultado de 5 ou mais pontos diagnostica depressão, sendo que o escore igual ou maior que 11 caracteriza depressão grave..20,21

Os voluntários foram submetidos ainda à avaliação da Força de Preensão Manual (FPM), como um componente da fragilidade física, medida pelo dinamômetro da marca Jamar, devidamente calibrado, realizada na mão dominante e não dominante. O idoso ficou sentado, o membro superior em teste foi posicionado: ombro em adução, articulação do cotovelo flexionado a $90^{\circ}$ e antebraço na posição neutra. O colaborador realizou três preensões intercaladas por tempo de 1 minuto e foi observada a média dessas três medidas, procedimento pelo qual é recomendado pela Sociedade Americana de Terapia da Mão (SATM) ${ }^{22}$ e Figueiredo, ${ }^{23}$ considerada a forma mais confiável de se medir a FPM ${ }^{24}$ e, ainda, essa média foi utilizada com base na mão dominante e os pontos de corte separados por sexo e idade. conforme protocolo. ${ }^{25}$

Todos os 150 colaboradores e/ou seus acompanhantes foram informados antes sobre os procedimentos e concordaram em assinar o Termo de Consentimento Livre e Esclarecido (TCLE).

\section{Análise estatística}

Para a estruturação do banco de dados, utilizou-se o aplicativo Microsoft Excel 2013 e, para as análises, o programa estatístico Statistical Pachage for Social Sciences (SPSS), versão $(21,0)$, para Windows. Primeiramente, as variáveis contínuas foram testadas quanto à normalidade de suas distribuições por meio do teste de Kolmogorov-Smirnov. Aquelas que seguiram a distribuição de Gauss foram analisadas por meio de testes paramétricos e as demais por testes não paramétricos (especificados nas Tabelas de resultados). Em todas as análises, um nivel de significância de $5 \%$ foi considerado $(p=0,05)$. A fim de comparar os graus de fragilidade, avaliados pela EFS, com a Idade e o Tempo de Internamento, o teste não paramétrico de Kruskal-Wallis foi aplicado. A comparação com a FPM foi feita por meio do teste paramétrico ANOVA. Após observada diferença significante estatisticamente na FPM, conforme o grau de fragilidade, foi dada sequência na análise Post Hoc por meio do teste LSD, a fim de comparar os grupos dois a dois e identificar as diferenças. O teste Qui-quadrado foi utilizado para avaliar a associação entre a fragilidade (conforme as categorias da EFS) com - Sexo, a Prática de Atividade Física, a Depressão e a Escolaridade. O Coeficiente de correlação de Spearman foi aplicado para investigar a presença de correlação entre o Tempo de Internamento com a FPM e a Idade.

\section{Resultados}

Os dados sociodemográficos da população estudada podem ser vistos na Tabela 1 por meio de média e desvio padrão ou frequência absoluta e percentual relativo. 
TABELA 1 - Caracteristicas gerais da amostra

\begin{tabular}{|c|c|c|}
\hline \multicolumn{2}{|c|}{ Variáveis } & \multirow{2}{*}{$\begin{array}{c}\begin{array}{c}\text { Média } \\
( \pm D P)\end{array} \\
\text { Freq (\%) }\end{array}$} \\
\hline Idade (anos) & & \\
\hline \multirow{4}{*}{$\begin{array}{l}\text { Idade Categórica } \\
\text { (anos) }\end{array}$} & 60 a 64 & $47(31,3)$ \\
\hline & 65 a 69 & $38(25,3)$ \\
\hline & 70 a 74 & $28(18,7)$ \\
\hline & $\geq 75$ & $37(24,7)$ \\
\hline \multirow{2}{*}{ Sexo } & Fem & $58(38,7)$ \\
\hline & Masc & $92(61,3)$ \\
\hline \multirow{4}{*}{ Estado Civil } & Solteiro & $13(8,7)$ \\
\hline & Casado & $90(60,0)$ \\
\hline & Divorciado & $16(10,7)$ \\
\hline & Viúvo & $31(20,7)$ \\
\hline \multirow{2}{*}{$\begin{array}{l}\text { Escolaridades } \\
\text { (anos) }\end{array}$} & o a 4 & $98(65.3)$ \\
\hline & $\geq 5$ & $52(34,7)$ \\
\hline \multirow{2}{*}{$\begin{array}{c}\text { Prática de } \\
\text { Atividade Física }\end{array}$} & Sim & $41(27,3)$ \\
\hline & Não & $109(72,7)$ \\
\hline \multirow{2}{*}{$\begin{array}{c}\text { Motivo do } \\
\text { Internamento }\end{array}$} & Pós Trauma & $15(10)$ \\
\hline & Clínico & $135(90)$ \\
\hline Tipo de & Cirúrgico & $69(46)$ \\
\hline Tratamento & Conservador & $81(54)$ \\
\hline $\begin{array}{c}\text { Tempo de } \\
\text { Internamento } \\
\text { (dias) }\end{array}$ & & $5,3 \pm 4,2$ \\
\hline
\end{tabular}

Dos 150 colaboradores, 61,3\% foram identificados como sendo do sexo masculino. A idade média dos colaboradores foi de 70 anos, com a maior frequência entre 60 e 64 anos (31,3\%). A grande maioria dos idosos era de casados (60\%), com baixa escolaridade (65.3\% com o a 4 anos) e sedentários (72,7\%). Do total, 90\% internaram por alguma causa de origem clínica, e apenas 10\% eram após trauma. Em 54\% dos casos o tratamento conservador foi elencado, enquanto o tratamento cirúrgico foi necessário para $46 \%$ dos sujeitos. 0 tempo médio de internamento foi de 5,3 dias $( \pm 4,2)$.

A Tabela 2 apresenta a comparação das variáveis Idade, Tempo de Internamento e FPM entre os graus de fragilidade, descritas por meio de média e desvio padrão (DP) com os respectivos valores de $p$.

TABELA 2 - Correlação dos graus de fragilidade da Escala de Edmonton com Idade, Tempo de Internamento e FPM

\begin{tabular}{|c|c|c|c|c|c|c|}
\hline \multirow{2}{*}{ Variáveis } & \multicolumn{5}{|c|}{ Escala de Edmonton } & \multirow[b]{2}{*}{$P$} \\
\hline & Normal & Vulnerável & $\begin{array}{l}\text { Fragilidade } \\
\text { Leve }\end{array}$ & $\begin{array}{l}\text { Fragilidade } \\
\text { Moderada }\end{array}$ & $\begin{array}{c}\text { Fragilidade } \\
\text { Grave }\end{array}$ & \\
\hline Idade (anos) & $67.5 \pm 5.5$ & $68,2 \pm 6,9$ & $70,2 \pm 7,5$ & $71,9 \pm 9,3$ & $73,2 \pm 9,2$ & $0,174^{1}$ \\
\hline $\begin{array}{c}\text { Tempo de } \\
\text { Internamento } \\
\text { (dias) }\end{array}$ & $3,81 \pm 2,5$ & $4,3 \pm 2,4$ & $5,8 \pm 4,0$ & $7,0 \pm 6,1$ & $5,0 \pm 4,5$ & 0,083 \\
\hline FPM (kgf) & $37,0 \pm 11,7$ & $31,2 \pm 11,0$ & $28,9 \pm 9,5$ & $24,1 \pm 10,0$ & $26,1 \pm 9,4$ & $0,001^{2}$ \\
\hline
\end{tabular}

FPM: Força de Preensão Manual; ${ }^{1}$ Teste de Kruskal-Wallis; ${ }^{2}$ Teste ANOVA; "Significância estatística $(p=0,05)$ 
Com relação à Escala de Edmonton, os participantes do estudo, 16 (10,7\%) foram classificados como "Normais", 45 (30\%) como "Vulneráveis", 41 (27,3\%) com "Fragilidade Leve", 34 (22,7\%) com "Fragilidade Moderada" e 14 (9,3\%) com "Fragilidade Grave". Conforme pode ser observado na Tabela 2 apenas a FPM apresentou diferença significante estatisticamente entre os graus de fragilidade.

A Tabela 3 apresenta a comparação da fragilidade, conforme a Escala de Edmonton, com relação ao Sexo, à Prática de Atividade Fisica, à Depressão e à Escolaridade, por meio de frequências e porcentagens, com os respectivos valores de $p$.

TABELA 3 - Correlação dos graus de fragilidade da Escala de Edmonton com relação ao sexo, prática de atividade fisica, depressão e escolaridade

\begin{tabular}{|c|c|c|c|c|c|c|c|}
\hline \multirow{2}{*}{\multicolumn{2}{|c|}{ Variáveis }} & \multicolumn{5}{|c|}{ Escala de Edmonton (Freq (\%)) } & \multirow[b]{2}{*}{$P$} \\
\hline & & Normal & Vulnerável & $\begin{array}{l}\text { Fragilidade } \\
\text { Leve }\end{array}$ & $\begin{array}{l}\text { Fragilidade } \\
\text { Moderada }\end{array}$ & $\begin{array}{c}\text { Fragilidade } \\
\text { Grave }\end{array}$ & \\
\hline \multirow{3}{*}{ Sexo } & Fem & $6(10,3)$ & $17(29,3)$ & $14(24,1)$ & $17(29,3)$ & $4(6,9)$ & \multirow{3}{*}{0,588} \\
\hline & & & & & & & \\
\hline & Masc & $10(10,9)$ & $28(30,4)$ & $27(29,3)$ & $17(18,5)$ & $10(10,9)$ & \\
\hline \multirow{2}{*}{$\begin{array}{c}\text { Prática de } \\
\text { Atividade Física }\end{array}$} & Sim & $8(19,5)$ & $10(24,4)$ & $15(36,6)$ & $6(14,6)$ & $2(4,9)$ & \multirow{2}{*}{0,054} \\
\hline & Não & $8(7,3)$ & $35(32,1)$ & $26(23,9)$ & $28(25,7)$ & $12(11,0)$ & \\
\hline \multirow{3}{*}{ Depressão } & Sim & $1(2,4)$ & $11(26,2)$ & $10(23,8)$ & $12(28,6)$ & $8(19,0)$ & \multirow{3}{*}{$0,025^{*}$} \\
\hline & & & & & & & \\
\hline & Não & $15(13,9)$ & $34(31,5)$ & $31(28,7)$ & $22(20,4)$ & $6(5,6)$ & \\
\hline \multirow[b]{2}{*}{ Escolaridade } & o a 4 anos & $7(7,1)$ & $25(25,5)$ & $31(31,6)$ & $25(25,5)$ & $10(10,2)$ & \multirow[b]{2}{*}{0,080} \\
\hline & $\begin{array}{c}5 \text { anos ou } \\
\text { mais }\end{array}$ & $9(17,3)$ & $20(38,5)$ & $10(19,2)$ & $9(17,3)$ & $4(7,7)$ & \\
\hline
\end{tabular}

*Significância estatística $(p=0,05)$; Teste Qui-quadrado

$\mathrm{Na}$ análise das graduações de fragilidade, avaliadas pela Escala de Edmonton, não foram observadas associações estatisticamente significativas de Sexo e Escolaridade. Em relação à Prática de Atividade Física, apesar da ausência de significância estatística, os resultados devem ser valorizados, visto que o valor de $p$ foi limitrofe. Conforme pode ser observado na Tabela 3. a frequência de participantes classificados como vulneráveis ou frágeis foi maior entre os não praticantes em comparação aos praticantes de atividade física. A Depressão foi a única que apresentou associação significativa com o grau de fragilidade. Dos 42 individuos identificados como depressivos, $71,4 \%$ foram classificados como frágeis (fragilidade leve, moderada e grave).

A Figura 1 apresenta os diagramas de caixa com a distribuição dos valores de FPM, conforme o Sexo (Figura 1 - A) e a Idade (Figura 1 - B). 
Figura 1 - Comparação da FPM com o Sexo e a Idade

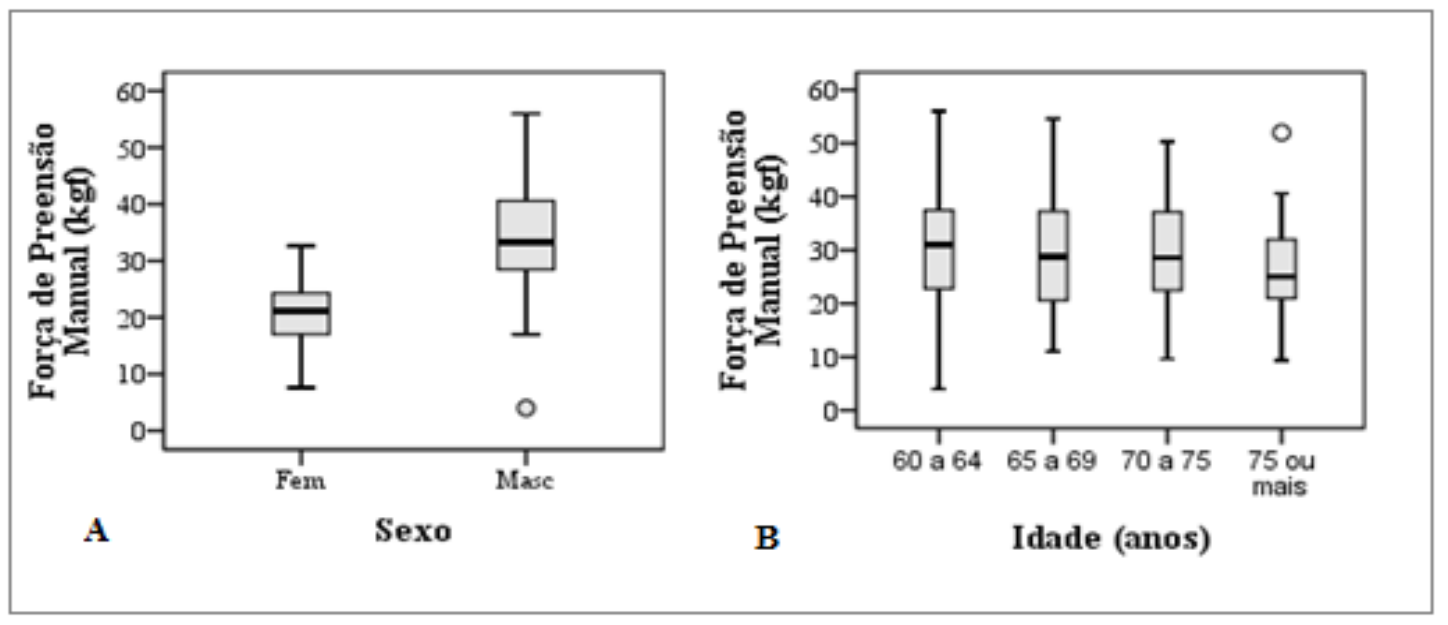

A - Distribuição dos valores de força de preensão manual conforme o sexo. Teste t de Student para Amostras Independentes $(p=0,000$ ); B - Distribuição dos valores de força de preensão manual conforme as categorias de idade. Teste ANOVA um fator $(p=0,295)$.

A FPM média entre as mulheres foi de 20,6 $\pm 6,1$ (mediana 21,2 (7,6 - 32,6)), enquanto entre os homens foi de $34,5 \pm 9,8$ (mediana 33,3 (4,0 - 56,0), diferença significante estatisticamente, com $p=0,000$. Já em relação à categoria idade, não foram observadas diferenças significativas entre os valores de FPM, cujo $p=0,295$ (60 a 65 anos: média 30,2 \pm 11,8, mediana 31,0 (4,0 - 56,0); 65 a 69 anos: média 30,3 $\pm 11,4$, mediana 28,8 (11,0 - 54,6); 70 a 75 anos: média 29,5 £ 10,9, mediana 28,6 (9,6 - 50,3); 75 anos ou mais: média 26,2 \pm 8,8, mediana $25,0(9,3-52,0)$.

\section{Discussão}

A idade dos idosos variou de 60 a 99 anos, com uma média de idade próximo ao que se encontra em outro estudo realizado com idosos frágeis internados (73,8 \pm 8,8), encontrados com maior frequência entre 60 e 69 anos. ${ }^{19,26}$ Quanto ao nível de escolaridade, 90 (65.3\%) possuíam entre zero e quatro anos de estudo, sendo eles analfabetos ou com ensino fundamental incompleto, resultados esses que também chamaram atenção no estudo de outro autor, ${ }^{19}$ pois baixos niveis de escolaridade predispõe idosos à maiores riscos de apresentarem problemas de saúde e, como consequência, um declínio relacionado à funcionalidade. ${ }^{27}$
A respeito da SF em instituições hospitalares, este estudo obteve como resultado dentro da EFS uma maior frequência de idosos "Vulneráveis", oposto ao que se encontra em outro estudo aplicado em ambiente hospitalar, em que há maior frequência de idosos classificados com "Fragilidade leve" pela EFS. ${ }^{26}$ Ainda, no presente estudo, observa-se menor frequência de idosos classificados como "Normais", resultado esse que corrobora com outro estudo. ${ }^{26}$

Talvez não haja uma explicação coerente para isso, porém essa variação em relação aos aspectos da classificação de fragilidade intra-hospitalar encontra a resposta no próprio processo hospitalização, fato que influencia os resultados desse estudo e chama atenção para a condição vulnerável do idoso que, muitas vezes, é acompanhada por um declínio irreversivel do estado funcional e por mudanças na qualidade e no estilo de vida. ${ }^{28,29}$

Estudos demonstram que há correlação entre aumento de idade e fragilidade, ${ }^{8,30,31}$ entretanto observa-se que há um valor crescente na idade dos idosos frágeis deste estudo, porém esses valores não apresentam resultados significativos, semelhante aos achados em ambiente hospitalar de outro autor. ${ }^{32}$

A fragilidade caracteriza-se por sinais e sintomas, dentre eles a diminuição da preensão pal- 
mar. ${ }^{33}$ Estudos ainda identificaram essa diminuição como sendo a mais frequentemente usada no diagnóstico da SF. ${ }^{34}$ Nesse estudo a diminuição da FPM realmente se caracterizou presente em idosos frágeis, resultados esses que igualmente são demonstrados em outros estudos. ${ }^{31,35}$

Há autores que relatam uma associação entre essa redução da força de preensão palmar com o sexo feminino, ${ }^{36,37}$ o que também foi possivel notar nesse estudo, resultados esses que podem ser explicados pelas mulheres apresentarem menor reserva de massa muscular que os homens. Convém ressaltar ainda, que a prevalência de baixa força de preensão foi maior entre as mais velhas, (80 anos ou mais) assim como no estudo de Garcia et al., ${ }^{38}$ corroborando com Ribeiro e Neri. ${ }^{39}$

Há relatos de que a correlação da fragilidade com o gênero, em que há prevalência maior em mulheres do que em homens, ${ }^{5,40,41,42}$ diferentemente, do que foi encontrado nos dados desse estudo e nos de outros autores. . $23,35,43$ Possivelmente esse evento ocorra porque os homens cuidam menos de sua saúde, quando comparados às mulheres e também por apresentar um maior indice de homens internados.

Idosos frágeis apresentam 80\% mais chances para o desenvolvimento da depressão, ${ }^{44}$ estima-se ainda que ela seja frequente em idosos hospitalizados. ${ }^{45} \mathrm{Um}$ estudo realizado na enfermaria geriátrica de um hospital ${ }^{46}$ relaciona a depressão com a prevalência da fragilidade, trazendo resultados com significância estatística. O presente estudo corrobora com o estudo citado.

Em outro estudo, ${ }^{47}$ os idosos frágeis apresentaram baixa prática de atividade física, já no presente estudo, mesmo apresentando ausência significativa, pode-se notar que a maioria daqueles classificados como vulneráveis ou frágeis não eram praticantes de atividade física.

A maioria dos idosos acima de 60 anos possui as variáveis baixa escolaridade e fragilidade associadas. 42 No presente estudo, a baixa escolaridade associada à fragilidade não obteve resultados significativos, condizente com o estudo nacional de idosos longevos.48.

A União Europeia tem dado uma importância especial às pessoas com fragilidade, como sendo grandes consumidoras dos serviços de saúde, com elevado risco de internamento em hospitais. ${ }^{49}$ Porém, não há estudos comparativos especíicos sobre os graus de fragilidade e o tempo de internamento, o que limitou a abordagem desse estudo, mesmo com resultados pouco significativos nessa comparação. No entanto, pode-se observar que a fragilidade é presente em idosos hospitalizados, então sugere-se a realização de novas pesquisas que busquem uma correlação mais aprofundada, com um enfoque mais direcionado.

\section{Conclusão}

A percepção da multidimensionalidade presente na coexistência entre baixos niveis de força muscular e certo grau de manutenção de padrões funcionais em idosos hospitalizados motivou a elaboração deste estudo.

No presente estudo, o comportamento sedentário trouxe à luz da ciência alguns fatores biológicos associados ao avançar da idade, correlacionando-os às variáveis socioeconômicas, que limitam o acesso à saúde, podendo prejudicar a qualidade de vida dos idosos. Dessa forma, caracterizar a funcionalidade deve ressaltar padrões de independência funcional no intuito da prevenção, a fim de diminuir os efeitos deletérios da redução do desempenho funcional e do processo de envelhecimento sobre seu bem-estar geral.

Consideramos que esse estudo foi importante. pois caracterizou idosos hospitalizados como uma população vulnerável e de risco, sugerindo que novas investigações enfoquem a importância e o cuidado do idoso para um melhor delineamento em uma visão interdisciplinar.

\section{Referências}

1. Dawalibi NW, Anacleto GMC, Witter C, Goulart RMM, Aquino RC. Envelhecimento e qualidade da vida: análise da produção cientifica da SciELO. Estud Psicol. 2013 Jan;30(3):393-403. https://doi.org/10.1590/S0103166X2013000300009.

2. Fried LP, Tangen CM, Walston J, Newman AB, Hirsch C, Gottdiener J, Seeman T, Tracy R, Kop WJ, Burke G, Mc Burnie MA. Frailty in older adults: evidence for a phenotype. J Gerontol A Biol Sci Med Sci 2001; 56(3):M146-M156. https://doi.org/10.1093/gerona/56.3.M146. 
3. LEME, Daniel Eduardo da Cunha et al. Estudo do impacto da fragilidade, multimorbidade e incapacidade funcional na sobrevida de idosos ambulatoriais. Ciênc Saúde Colet. 2019;24(1):137-46. https://doi. org/10.1590/1413-81232018241.04952017.

4. Fron JRS, Rosset I, Freitas CP, Silva AO, Santos JLF, Rodrigues RAP. Prevalência de quedas em situações de fragilidade. Rev Saúde Pública. 2013:47(2):266-73. https://doi.org/10.1590/S0034-8910.2013047003468.

5. Certo AC, Sanchez K, Galvão A, Fernandes H. A síndrome da fragilidade nos idosos: revisão da literatura. Rev. Acta de Gerontologia. 2016;2(1):1-11.

6. Brasil. DATASUS. Informações de Saúde Demográficas e Socioeconômicas. Disponivel em: 2010.

7. Veras RP, Oliveira MR. Linha de cuidado para o idoso: detalhando o modelo. Rev. Bras. Geriatr. Gerontol 2016; 19(6):887-905. https://doi.org/10.1590/198122562016019.160205.

8. Marchiori GF. Prevalência e preditores da sindrome fragilidade em idosos durante e após hospitalização. Dissertação (Mestrado em Atenção à Saúde) - Universidade Federal do Triângulo Mineiro, Uberaba, 2015.

9. Chaimowicz F. Saúde do Idoso. $2^{\mathrm{a}}$ ed. Belo Horizonte (MG): NESCON-UFMG; 2013.

10. Nunes BP, Soares MU, Wachs LS, Volz PM, Saes MO, Duro SMS, et al. Hospitalization in older adults: association with multimorbidity, primary health care and private health plan. Rev. saúde pública [Online]. 2017:51(43). Doi: http://dx.doi.org/10.1590/S1518-8787.2017051006646.

11. Fortin M, Stewart M, Poitras ME, Almirall J, Maddocks $H$. A systematic review of prevalence studies on multimorbidity: toward a more uniform methodology. Ann Fam Med. 2012;10(2):142-51. https://doi.org/10.1370/afm.1337.

12. Lehnert T, Heider D, Leicht $H$, Heinrich S, Corrieri S, Luppa $M$, et al. Review: health care utilization and costs of elderly persons with multiple chronic conditions. Med Care Res Rev. 2011;68(4):387-420. https:// doi.org/10.1177/1077558711399580.

13. Veras RP, Caldas CP, Motta LB, Lima KC, Siqueira RC, Rodrigues RTSV et al. Integration and continuity of Care in health care network models for frail older adults. Rev Saude Publica. 2014:48(2):357-65. https:// doi.org/10.1590/S0034-8910.2014048004941.

14. Marengoni A, Angleman S, Melis R, Mangialasche F, Karp A, Garmen A, et al. Aging with multimorbidity: a systematic review of the literature. Ageing Res Rev. 2011;10(4):430-9. https://doi.org/10.1016/j.arr.2011.03.003.

15. Harrison C, Britt H, Miller G, Henderson J. Examining different measures of multimorbidity, using a large prospective cross-sectional study in Australian general practice. BMJ Open. 2014;4(7):e004694. https://doi. org/10.1136/bmjopen-2013-004694.

16. Gil AC. Como elaborar projetos de pesquisa. $6^{\circ} \mathrm{ed}$. São Paulo: Atlas. 2017.
17. Brucki SMD, Nitrini R, Caramelli P, Bertolucci PHF, Okamoto $\mathrm{IH}$. Suggestions for utilization of the mini-mental state examination in Brazil. Arq Neuropsiquiatr 2003: 61(3B):777-781. https://doi.org/10.1590/S0004282X2003000500014.

18. Fabricio-Wehbe SCC, Schiaveto FV, Vendrusculo TRP, Haas VJ, Dantas RAS, Rodrigues RAP. Cross-cultural adaptation and validity of the "Edmonton Frail Scale EFS" in a Brazilian elderly sample. Rev Latino-Am Enfermagem [lnternet]. 2009 [cited 2014 Mar 31];17(6):1043-9. https://doi.org/10.1590/S0104-11692009000600018.

19. Yesavage JA, Brink TL Rose TL et al. Development and validation of a geriatric depression screening scale: a preliminary report. J Psychiat Res. 1983;17:37-49. https://doi.org/10.1016/0022-3956(82)90033-4..

20. Almeida OP, Almeida SA. Confiabilidade da versão brasileira da Escala de Depressão em Geriatria (GDS) versão reduzida. Arq Neuro-Psiquiatr. 1999;57:421-6. https://doi.org/10.1590/S0004-282X1999000300013.

21. Frank MH, Rodrigues NL. Depressão, ansiedade, outros distúrbios afetivos e suicídio. In: Freitas EV, Py L, Neri AL, et al. Tratado de geriatria e gerontologia. $2^{a}$ ed. Rio de Janeiro: Guanabara; 2006; 376-87.

22. Fess EE. Grip strength. In: Casanova JS. Clinical Assessment Recommendations. 2nd ed. Chicago: American Society of Hand Therapists, 1992:41-45.

23. Figueiredo IM, Sampaio RF, Mancini MC, Silva FCM, Souza MAP. Test of grip strength using the Jamar dynamometer. Acta Fisiatr. 2007:14(2):104-10. https://doi. org/10.5935/0104-7795.20070002.

24. Stratford PW. Summarizing the results of multiple strength trials: truth or consequence. Physio Can. 1992;44:14-8.

25. Rosa TEC, Benicio MHD, Latorre MRDO, Ramos LR Fatores determinantes da capacidade funcional entre idosos. Rev Saúde Pública. 2003 Fev; 37(1):40-8. https:// doi.org/10.1590/S0034-89102003000100008.

26. Morton C. Creditor, MD. Hazards of hospitalization of the elderly. Ann Intern Med. 1993 Feb;118(3):219-23. https:// doi.org/10.7326/0003-4819-118-3-199302010-00011.

27. Herring, J. Vulnerable adults and the law. Oxford: Oxford University, 2016. https://doi.org/10.1093/acprof:0so/9780198737278.001.0001.

28. Bohannon RW, Peolsson A, Massy-Westropp N, Desrosiers J, Bear-Lehman J. Reference values for adult grip strength measured with a Jamar dynamometer: a descriptive meta-analysis. Physiotherapy, 2006:92:11-15. https://doi.org/10.1016/j.physio.2005.05.003.

29. Storti LB, Fabrício-Whebe SCC, Kusumota L, Rodrigues RAP, Marques S. Fragilidade de idosos internados na clínica médica da unidade de emergência de um hospital geral terciário. Texto contexto Enferm. 2013:22(2):452-9. https://doi.org/10.1590/S010407072013000200022 .

30. Remor CB, Bós AJG, Werlang MC. Características relacionadas ao perfil de fragilidade no idoso. Sci Med 2011;21(3):107-12. 
31. Fernandes LC, Fernandes VLS, Costa MN, Siqueira A, Menezes RL. Idosos institucionalizados: frágeis e sem equilibrio. Rev. Educ. Saúde. 2016;4(2):95-102.

32. Freitas CV, Sorges ESNF, Moreira KECS, Carneiro SR. Avaliação de fragilidade, capacidade funcional e qualidade de vida dos idosos atendidos no ambulatório de geriatria de um hospital universitário. Rev Bras Geriatr Gerontol. 2016. 19(1):119-128.

33. Lana LD, Schneider RH. Sindrome De fragilidade no idoso: uma revisão narrativa. Rev Brasileira Geriatr Gerontol. 2014; 17(3):673-680. https://doi. org/10.1590/1809-9823.2014.12162.

34. Carmo LV, Drummond LP, Arantes PMM. Avaliação do nivel de fragilidade em idosos participantes de um grupo de convivência. Fisioter Pesqui. 2011;18(1):17-22. https://doi.org/10.1590/S1809-29502011000100004.

35. Oliveira DR, Bettinelli LA, Pasqualotti A, Corso D, Brock F, Erdmann AL. Prevalência de sindrome em idosos de uma instituição hospitalar. Rev. Latino-Am. Enfermagem. 2013;21(4):1-8. https://doi.org/10.15.90/ S0104-11692013000400009.

36. Lenardt MH, Grden CRB, Sousa JAV, Reche PM, Betiolli SE, Ribeiro DKMN. Fatores associados a diminuição de força de preensão manual em idosos longevos. Rev Esc Enferm USP. 2014;48(6):1006-12. https://doi. org/10.1590/S0080-623420140000700007.

37. Silva NA, Menezes TN, Melo TLP, Pedraza DF. Força de preensão manual e flexibilidade e suas relações com variáveis antropométricas em idosos. Rev Assoc Med Bras. 2013:59(2):128-35. https://doi.org/10.1016/j. ramb.2012.10.002.

38. Garcia PA, Dias JMD, Dias RC, Zampa CC. Estudo da relação entre função muscular, mobilidade funcional e nivel de atividade física em idosos comunitários. Rev Bras Fisioter 2011; 15(1):15-22. https://doi.org/10.1590/ S1413-35552011010200001.

39. Ribeiro LHM, Neri AL. Exercicios fisicos, força muscular e atividades de vida diária em mulheres idosas. Cienc Saúde Coletiva. 2012;17(8):2169-80. https://doi. org/10.1590/S1413-81232012000800027.

40. Tribes S, Oliveira RJ. Síndrome da fragilidade biológica em idosos: revisão sistemática. Rev Salud Pública. 2011;13(5): 853-864. https://doi.org/10.1590/S012400642011000500014.

41. Fhon JRS, Diniz MA, Leonardo KC, Kusumota L, Haas VJ, Rodrigues RAP. Sindrome da fragilidade relacionada a incapacidade funcional no idoso. Revista Acta Paul Enferm. 2012;00(0):000-0.

42. Castell MV, Sánchez M, Julián R, Queipo R, Martín S, Otero Á. Prevalência de fraqueza e velocidade de caminhada lenta em pessoas de 65 anos ou mais: implicações para cuidados primários. BMC family practice. 2013;14(86):74-86. https://doi.org/10.1186/1471-2296-14-86.

43. Lenardt MH, Carneiro NHK, Binotto MA, Setoguchi LS, Cechinel C. Relação entre fragilidade física e características sociodemográficas e clínicas de idosos. Esc. Anna Nery. 2015:19(4):585-92. https://doi. org/10.5935/1414-8145.20150078.
44. Pegorari MS, Tavares DMS. Fatores associados à sindrome da fragilidade em idosos residentes em uma área urbana. Rev. Latino-Am. Enfermagem. 2014; 22(5):874-82. https://doi.org/10.1590/0104-1169.0213.2493.

45. Roesler E, Resende A, Sousa P, Ferreira LB. Prevalência e fatores associados à depressão entre idosos institucionalizados: susbsídio ao cuidado de enfermagem. Rev Esc Enferm USP. 2012;46(6):1387-93. https:// doi.org/10.1590/S0080-62342012000600015.

46. Joosten E, Demuynck M, Detroyer E, Melisen K. Prevalência de fragilidade e sua capacidade de prever no delírio hospitalar, quedas e mortalidade de 6 meses em pacientes idosos hospitalizados. BMC Geriatr. 2014:14(1):000-0.

47. Nunes DP, Duarte YAO, Santos JLF, Lebrão ML. Rastreamento de fragilidade em idosos por instrumento autorreferido. Rev Saúde Pública. 2015:49(2):1-9. https:// doi.org/10.1590/S0034-8910.2015049005516.

48. Grden CRB, Lenardt MH, Sousa JAV, Kusomota L, Dellaroza MSG. Betiolli SE. Associação da síndrome da fragilidade fisica às características sociodemográficas de idosos longevos da comunidade. Rev Latino-Am Enfermagem. 2017:25:e2886.

49. Pinto MJCP, Coutinho SCS. Síndrome da Fragilidade Frailty sindrome. International Journal of Developmentel and Educational Psychology. Rev De Psicologia. 2014:2(1):171-6. https://doi.org/10.17060/ijodaep/2014. n2.v1.020.

\section{Luciano Alves Leandro}

Doutorando em Medicina Interna pela Pontificia Universidade Católica do Paraná (PUCPR), em Curitiba, PR, Brasil.

\section{Laryssa Mireli Rodrigues Gomes}

Residente de Fisioterapia em Terapia Intensiva pela Pontificia Universidade Católica do Paraná (PUCPR), em Curitiba, PR, Brasil.

\section{Juliane Pessoa Chevônica}

Especializanda em Acupuntura Tradicional Chinesa pela Pontificia Universidade Católica do Paraná (PUCPR), em Curitiba, PR, Brasil.

\section{Endereço para correspondência}

Luciano Alves Leandro

Pontifícia Universidade Católica do Paraná

Clínica Escola de Fisioterapia e Reabilitação - PUCPR

Av. Sen. Salgado Filho, 555

Prado Velho, 80215270

Curitiba, PR, Brasil 